\title{
A New Method for Computing Elimination Ideals of Likelihood Equations
}

\author{
Xiaoxian Tang* \\ School of Mathematics and Systems \\ Science, \\ Beihang University \\ Beijing, China \\ xiaoxian@math.tamu.edu
}

\author{
Timo de Wolff \\ Department für Mathematik, \\ Technische Universität Braunschweig \\ Braunschweig, Germany \\ t.de-wolff@tu-braunschweig.de
}

\author{
Rukai Zhao \\ Computer Science \& Engineering, \\ Texas A\&M University \\ College Station, Texas \\ zhaorukai@tamu.edu
}

\begin{abstract}
We develop a probabilistic algorithm for computing elimination ideals of likelihood equations. We show experimentally that it is far more efficient than directly computing Gröbner bases or the interpolation method proposed in $[39,40]$ for medium to large size models. Furthermore, we deduce discriminants of the elimination ideals, which play a central role in real root classification. In particular, we can compute the discriminant of one Jukes-Cantor model in phylogenetics (with size 8 GB text file).
\end{abstract}

\section{CCS CONCEPTS}

- Computing methodologies $\rightarrow$ Symbolic and algebraic manipulation; Algebraic algorithms.

\section{KEYWORDS}

Maximum likelihood estimation, Likelihood equation, Real root classification, Discriminant, Elimination ideal

\section{ACM Reference Format:}

Xiaoxian Tang, Timo de Wolff, and Rukai Zhao. 2019. A New Method for Computing Elimination Ideals of Likelihood Equations. In International Symposium on Symbolic and Algebraic Computation (ISSAC '19), fuly 15-18, 2019, Beijing, China. ACM, New York, NY, USA, 8 pages. https://doi.org/10. $1145 / 3326229.3326241$

\section{INTRODUCTION}

This work is motivated by the maximum likelihood estimation problem in statistics:

Which probability distribution describes a given data set optimally for a chosen statistical model?

A standard way to answer this question is to determine a point in the model that maximizes a likelihood function; see (1). When the model is algebraic (see Definition 2.2) and the data is discrete, then one finds all critical points of the likelihood function by solving a system of likelihood equations (2) via applying Lagrange multipliers.

${ }^{*}$ Corresponding author

Permission to make digital or hard copies of all or part of this work for personal or classroom use is granted without fee provided that copies are not made or distributed for profit or commercial advantage and that copies bear this notice and the full citation on the first page. Copyrights for components of this work owned by others than ACM must be honored. Abstracting with credit is permitted. To copy otherwise, or republish, to post on servers or to redistribute to lists, requires prior specific permission and/or a fee. Request permissions from permissions@acm.org.

ISSAC '19, fuly 15-18, 2019, Beijing, China

(c) 2019 Association for Computing Machinery.

ACM ISBN 978-1-4503-6084-5/19/07 . \$ \$15.00

https://doi.org/10.1145/3326229.3326241
Solving likelihood equations motivates an important branch in algebraic statistics [1, 13, 14, 24-27, 31].

Likelihood equations form an algebraic system in probability variables $p_{0}, \ldots, p_{n}$, Lagrange multipliers $\lambda_{1}, \ldots, \lambda_{s+1}$, and parameters $u_{0}, \ldots, u_{n}$ representing the data obtained from statistical experiments. Given such a system with generically chosen data vector $\left(u_{0}, \ldots, u_{n}\right)$, the number of complex solutions is a finite non-negative constant, called the maximum-likelihood-degree (MLdegree); see Definition 2.5 and $[27,31]$. Since the variables $p_{i}$ represent probabilities, one is especially interested in a real solution classification $[15,33,47]$ of likelihood equations. Unfortunately, this classification is very challenging, since it is a specific real quantifier elimination problem $[2-5,7-11,16,17,23,28-30,34-38,41,42,45]$, a fundamental problem in computational real algebraic geometry.

The number of real solutions only changes when the parameters (data) pass a set called the discriminant variety; see [33, Definition 1]. Hence, these discriminant varieties of likelihood equations play a core role in real solution classification. In [40], Rodriguez and the first author studied how to compute discriminant varieties for a likelihood equation system efficiently. Experiments [40, Tables 2-3] suggest that Gröbner bases [12, 21, 22] cannot be computed directly for medium to large size models. Rodriguez and the first author proposed in [40, Algorithm 2] a probabilistic algorithm based on evaluation/interpolation techniques, which, in theory, works for arbitrary general zero-dimensional systems. In practice, however, it is limited to small algebraic models with ML-degrees not greater than 6 . The main bottleneck is that the size of the discriminants we are trying to compute are huge; for instance, a model with MLdegree 6 has a discriminant with total degree 12 and thousands terms.

The key idea of this article is to exploit the special structure of likelihood equations to both improve the computational efficiency and to allow ourselves to compute generators of elimination ideals instead of computing discriminants directly. We summarize the entire challenge in Figure 1. Thus, the task of this paper is to efficiently compute the elimination ideal with respect to all parameters (data) and one variable for a given system of likelihood equations. More precisely, we have the following problem statement: Input: Likelihood equations

$$
f_{0}, \ldots, f_{n+s+1} \in \mathbb{Q}\left[u_{0}, \ldots, u_{n}, p_{0}, \ldots, p_{n}, \lambda_{1}, \ldots, \lambda_{s+1}\right] .
$$

Output: A generator of the elimination ideal with respect to one probability variable $p_{i}$ for some $i$ between 0 and $n$

$$
\sqrt{\left\langle f_{0}, \ldots, f_{n+s+1}\right\rangle \cap \mathbb{Q}\left[u_{0}, \ldots, u_{n}, p_{i}\right]} .
$$




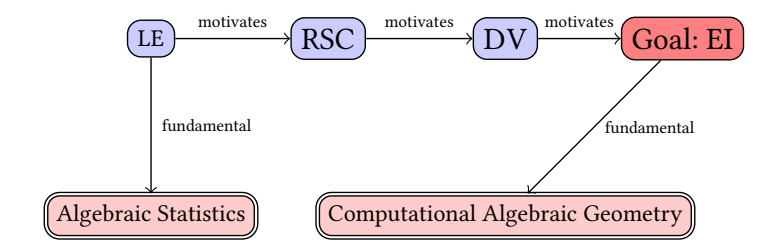

LE: Solving Likelihood Equations; $\quad$ RSC: Real Solution Classification; DV: Computing Discriminant Varieties; EI: Computing Elimination Ideals.

Figure 1: An visualization of our motivation.

\begin{tabular}{|c|c|c|c|c|c|}
\hline \multirow{2}{*}{ Models } & \multirow{2}{*}{$* p_{i}$} & \multirow{2}{*}{ MLD } & \multicolumn{4}{|c|}{ Timings } \\
\cline { 4 - 6 } & & & Standard & Interpolation & Algorithm 1 \\
\hline 1 & 4 & 3 & $\mathbf{0 . 0 4 6} \mathrm{s}$ & $1.831 \mathrm{~s}$ & $0.525 \mathrm{~s}$ \\
\hline 2 & 6 & 2 & $\mathbf{0 . 5 2 4} \mathrm{s}$ & $24.983 \mathrm{~s}$ & $2.310 \mathrm{~s}$ \\
\hline 3 & 6 & 4 & $\mathbf{3 . 2 1 1} \mathrm{s}$ & $282.425 \mathrm{~s}$ & $16.174 \mathrm{~s}$ \\
\hline 4 & 6 & 6 & $\infty$ & $7933.230 \mathrm{~s}$ & $\mathbf{7 8 2 . 6 7 6} \mathrm{s}$ \\
\hline 5 & 5 & 12 & $\infty$ & $10726.268 \mathrm{~s}$ & $\mathbf{7 6 1 . 2 5 7} \mathrm{s}$ \\
\hline 6 & 9 & 10 & $\infty$ & $>1583 \mathrm{~d}$ & $\mathbf{1 4} \mathrm{d}$ \\
\hline 7 & 5 & 23 & $\infty$ & $9919.260 \mathrm{~s}$ & $\mathbf{4 6 2 4 . 5 7 5} \mathrm{s}$ \\
\hline 8 & 8 & 14 & $\infty$ & $>4667 d$ & $>15 d$ \\
\hline 9 & 8 & 9 & $\infty$ & $>39 \mathrm{~d}$ & $2 \mathrm{~d}$ \\
\hline
\end{tabular}

Table 1: Runtimes for computing elimination ideals (s: seconds; d: days). The models are sorted by the same order with [40, Table 3]. The column "standard" constains the runtimes via a regular FGb Gröbner basis computation, the column "Interpolation" contains the runtimes for [40, Algorithm 2], and the last column contains the runtimes for our Algorithm 1.

Our main contribution is the development of a probabilistic algorithm, Algorithm 1, for computing elimination ideals of Lagrange likelihood equations. We implemented the algorithm in Maple. Our experiments, summarized in Table 1, show that Algorithm 1 is significantly more efficient than the standard approach of directly computing Gröbner bases, or the evaluation/interpolation method in [40] for statistical models beyond small size, see Table 1 and Section 6 for further details. Our crucial idea to save computational time is to exploit an observation that the elimination ideals have special structures; see (3).

In particular, we are able to compute the discriminant of one Jukes-Cantor model [27, $P_{\text {comb }}$, Example 15] in phylogenetics [44, Chapter 15]. This was impossible before. We point out that this is a gigantic polynomial, whose total degree is 176 , and which take several GB memory when stored in a text file, see Table 2 (Model 9) for further details.

The article is organized as follows. Section 2 are preliminaries. In Section 3, we discuss the specialization properties of (radical) elimination ideals and multivariate factorization. In Section 4, we introduce general zero-dimensional systems. In Section 5, we introduce Algorithm 1 together with a list of sub-algorithms for computing elimination ideals of likelihood equations. In Section 6, we explain the implementation details and compare the efficiency of our code with existing methods.

\section{PRELIMINARIES}

We assume that the reader is familiar with the fundamental concepts of computational algebraic geometry. For a general overview, we refer the reader to [18] and [43].

\subsection{Notation}

Throughout the paper, we use bold letters for vectors or a finite set of polynomials, e.g., $z=\left(z_{1}, \ldots, z_{n}\right)$ and $H=\left\{h_{1}, \ldots, h_{m}\right\}$. For $h \in \mathbb{Q}[z]$ we denote the total degree of $h$ by $\operatorname{deg}(h)$ and the degree of $f$ with respect to a particular variable $z_{j}$ as $\operatorname{deg}\left(h, z_{j}\right)$. We denote by $\operatorname{coeff}\left(h, z_{j}^{i}\right)$ the coefficient of $h$ with respect to the monomial $z_{j}^{i}$. If $N=\operatorname{deg}\left(h, z_{j}\right)$, then simply denote $\operatorname{coeff}\left(h, z_{j}^{N}\right)$ by lcoeff $\left(h, z_{j}\right)$. For $H \subseteq \mathbb{Q}[\boldsymbol{z}]$, we denote by $\langle\boldsymbol{H}\rangle$ the ideal generated by $\boldsymbol{H}$ in $\mathbb{Q}[\boldsymbol{z}]$, and by $\mathcal{V}(\boldsymbol{H})$ the affine variety $\left\{\boldsymbol{z} \in \mathbb{C}^{n} \mid h(z)=\right.$ 0 for all $h \in \boldsymbol{H}\}$. For any ideal $\mathcal{I} \subset \mathbb{Q}[z]$, we denote by $\sqrt{I}$ the radical ideal of $\mathcal{I}$, and we denote by $\mathcal{V}(\mathcal{I})$ the affine variety defined by the generator polynomials of $\mathcal{I}$. For any subset $\mathcal{S} \subseteq \mathbb{C}^{n}$, we denote by $\mathcal{I}(\mathcal{S})$ the ideal generated by the polynomials vanishing on $\mathcal{S}$, i.e., $\mathcal{I}(\mathcal{S})=\left\{h \in \mathbb{Q}[z] \mid h\left(z^{*}\right)=0\right.$ for all $\left.z^{*} \in \mathcal{S}\right\}$, and denote by $\overline{\mathcal{S}}$ the Zariski closure $\mathcal{V}\left(\mathcal{I}(\mathcal{S})\right.$ ) of $\mathcal{S}$ in $\mathbb{C}^{n}$. For a positive integer $n$, and for any $1 \leq i \leq n$, we denote the canonical projection by $\operatorname{proj}_{i}: \mathbb{C}^{n} \rightarrow \mathbb{C}^{i}$. Finally, we denote by $\operatorname{gcd}(a, b)$ the greatest common divisor of two integers $a, b$.

\subsection{Algebraic Statistics}

In this section we recall the basic notions from algebraic statistics, which we need in this article.

Definition 2.1 (Probability Simplex). We define the $n$-dimensional probability simplex as $\Delta_{n}=\left\{\left(p_{0}, \ldots, p_{n}\right) \in \mathbb{R}^{n+1} \mid p_{0}>\right.$ $\left.0, \ldots, p_{n}>0, p_{0}+\cdots+p_{n}=1\right\}$.

Definition 2.2 (Algebraic Statistical Model and Model Invariant). Given homogenous polynomials $g_{1}, \ldots, g_{s} \in \mathbb{Q}\left[p_{0}, \ldots, p_{n}\right]$ such that $\mathcal{V}\left(g_{1}, \ldots, g_{s}\right) \subsetneq \mathbb{C}^{n+1}$ is irreducible and generically reduced, we define an algebraic statistical model as

$$
\mathcal{M}=\mathcal{V}\left(g_{1}, \ldots, g_{s}\right) \cap \Delta_{n} .
$$

Each $g_{i}$ is called a model invariant of $\mathcal{M}$. If $\mathcal{V}\left(g_{1}, \ldots, g_{s}\right)$ has codimension $s$, then we say $\left\{g_{1}, \ldots, g_{s}\right\}$ is a set of independent model invariants.

Given an algebraic statistical model $\mathcal{M}$ and a data vector $\boldsymbol{u}=$ $\left(u_{0}, \ldots, u_{n}\right) \in \mathbb{R}_{\geq 0}^{n+1}$, the maximum likelihood estimation (MLE) problem is the optimization problem

$$
\max \Pi_{k=0}^{n} p_{k}^{u_{k}} \text { subject to } \boldsymbol{p} \in \mathcal{M},
$$

which is fundamental in statistics [20, Chapter 2]. One way to solve the MLE problem is to solve a system of likelihood equations [27] formulated by the Lagrange multiplier method. We give the explicit formulation of such a system:

Definition 2.3 (Lagrange Likelihood Equations). Given an algebraic statistical model $\mathcal{M}$ with a set of independent model invariants $\left\{g_{1}, \ldots, g_{s}\right\} \subseteq \mathbb{Q}\left[p_{0}, \ldots, p_{n}\right]$, the polynomial set $f=$ 
$\left\{f_{0}, \ldots, f_{n+s+1}\right\}$ below is said to be the system of Lagrange likelihood equations of $\mathcal{M}$ when set equal to zeros:

$$
\begin{aligned}
f_{0}(u, p, \lambda)= & p_{0} \cdot\left(\frac{\partial g_{1}}{\partial p_{0}} \lambda_{1}+\cdots+\frac{\partial g_{s}}{\partial p_{0}} \lambda_{s}+\lambda_{s+1}\right)-u_{0}, \\
& \vdots \\
f_{n}(u, p, \lambda)= & p_{n} \cdot\left(\frac{\partial g_{1}}{\partial p_{n}} \lambda_{1}+\cdots+\frac{\partial g_{s}}{\partial p_{n}} \lambda_{s}+\lambda_{s+1}\right)-u_{n}, \\
f_{n+1}(u, p, \lambda)= & g_{1}\left(p_{0}, \ldots, p_{n}\right), \\
& \vdots \\
f_{n+s}(u, p, \lambda)= & g_{s}\left(p_{0}, \ldots, p_{n}\right), \\
f_{n+s+1}(u, p, \lambda)= & g_{s+1}\left(p_{0}, \ldots, p_{n}\right):=p_{0}+\cdots+p_{n}-1,
\end{aligned}
$$

with inderterminates $u=\left(u_{0}, \ldots, u_{n}\right), p=\left(p_{0}, \ldots, p_{n}\right)$, and $\lambda=$ $\left(\lambda_{1}, \ldots, \lambda_{s+1}\right)$. More specifically, $u_{0}, \ldots, u_{n}$ are parameters, and $p_{0}, \ldots, p_{n}, \lambda_{1}, \ldots, \lambda_{s+1}$ are variables.

THeOREM 2.4. [27] Given a system of Lagrange likelihood equations $f_{0}, \ldots, f_{n+s+1}$ defined in (2), there exists an affine variety $V \subsetneq$ $\mathbb{C}^{n+1}$, and a non-negative integer $N$ such that for any $\boldsymbol{b} \in \mathbb{C}^{n+1} \backslash V$, the equations

$$
f_{0}(\boldsymbol{b}, \boldsymbol{p}, \boldsymbol{\lambda})=\cdots=f_{n+s+1}(\boldsymbol{b}, \boldsymbol{p}, \boldsymbol{\lambda})=0
$$

have $N$ common complex solutions in $\mathbb{C}^{n+1} \times \mathbb{C}^{s+1}$.

Definition 2.5 (Maximum-Likelihod-Degree). [27] Given an algebraic statistical model $\mathcal{M}$ with a system of Lagrange likelihood equations defined as in (2), the non-negative integer $N$ stated in Theorem 2.4 is called the maximum-likelihood-degree, short $M L$ degree, of $\mathcal{M}$.

Definition 2.6 (Mixed Discriminant). [40, Definition 4] Given an algebraic statistical model $\mathcal{M}$ with a system of Lagrange likelihood equations $f=\left\{f_{0}, \ldots, f_{n+s+1}\right\}$ defined in (2), we define

$$
\mathcal{L}_{M J}=\overline{\operatorname{proj}_{n+1}(\mathcal{V}(f) \cap \mathcal{V}(J))},
$$

where $J$ denotes the determinant of Jacobian matrix of $f$ with respect to $(\boldsymbol{p}, \boldsymbol{\lambda})$ :

$$
\operatorname{det}\left[\begin{array}{cccccc}
\frac{\partial f_{0}}{\partial p_{0}} & \ldots & \frac{\partial f_{0}}{\partial p_{n}} & \frac{\partial f_{0}}{\partial \lambda_{1}} & \ldots & \frac{\partial f_{0}}{\partial \lambda_{s+1}} \\
\vdots & \ddots & \vdots & \vdots & \ddots & \vdots \\
\frac{\partial f_{n+s+1}}{\partial p_{0}} & \cdots & \frac{\partial f_{n+s+1}}{\partial p_{n}} & \frac{\partial f_{n+s+1}}{\partial \lambda_{1}} & \cdots & \frac{\partial f_{n+s+1}}{\partial \lambda_{s+1}}
\end{array}\right] .
$$

If $\mathcal{I}\left(\mathcal{L}_{M J}\right)$ is principal, then a generator polynomial of $\mathcal{I}\left(\mathcal{L}_{M J}\right)$, denoted by $\mathcal{D}_{M J}$, is said to be a mixed discriminant of $f$.

It is important to highlight that $\mathcal{L}_{M J}$ is a component of the discriminant variety [33] of Lagrange likelihood equations, which plays a central role of real root classification; see e.g., [40, Theorem 2]. Notice that if $\mathcal{L}_{M J}$ is principal, then the mixed discriminant $\mathcal{D}_{M J}$ is homogenuous in $\mathbb{Q}[\boldsymbol{u}]$; see [40, Proposition 2]. By Definition 2.6, $\mathcal{D}_{M J}$ can be computed by computing the elimination ideal $\langle f, J\rangle \cap \mathbb{Q}[\boldsymbol{u}]$. That means to compute a Gröbner base of $\langle f, J\rangle$ with respect to a lexicographic order, where the determinant $J$ of the Jacobian matrix, defined as in Definition 2.6, can be huge. So, computing $\mathcal{D}_{M J}$ is usually challenging in practice; see [40, Tables $2-3]$.

According to [40, Algorithm 2, Strategy 3], one way to improve the efficiency for computing $\mathcal{D}_{M J}$ is to compute the elimination ideal with respect to one probability variable first; for instance, $\langle f\rangle \cap \mathbb{Q}\left[\boldsymbol{u}, p_{0}\right]$. If $\sqrt{\langle f\rangle \cap \mathbb{Q}\left[\boldsymbol{u}, p_{0}\right]}=\left\langle E_{f}\right\rangle$, then it is well known that $\mathcal{D}_{M J}$ is a factor of the discriminant of $E_{f}$ with respect to $p_{0}$; see
[40, Lemma 3]. However, $E_{f}$ is hard to obtain via directly computing Gröbner bases; see the column "standard" in Table 1. Therefore, the goal of the rest of paper is to compute $E_{f}$ more efficiently.

\section{SPECIALIZATION PROPERTIES}

In this section, we discuss a selection of specialization properties, which guarantee the correctness of our Algorithm 1 in Section 5. Roughly speaking, elimination ideals (and their radicals) "specialize well" over a Zariski open set (see Proposition 3.2), and frac In what follows, we consider polynomial rings with at least two variables, i.e., $\mathbb{Q}\left[z_{1}, \ldots, z_{n}\right]$ with $n \geq 2$. Given $h \in \mathbb{Q}\left[z_{1}, \ldots, z_{n}\right]$, we denote for every $1 \leq i<n$, by $\operatorname{lm}_{i}(h)$ and $\operatorname{lcoeff}_{i}(h)$ the leading monomial and leading coefficient of $h$ with respect to $z_{i+1}, \ldots, z_{n}$, when $h$ is considered in $\mathbb{Q}\left(z_{1}, \ldots, z_{i}\right)\left[z_{i+1}, \ldots, z_{n}\right]$ with the lexicographic or$\operatorname{der} z_{i+1}<\cdots<z_{n}$. For every $\boldsymbol{b}=\left(b_{1}, \ldots, b_{i}\right) \in \mathbb{C}^{i}$, we define the polynomial $h(\boldsymbol{b})=\left.h\right|_{z_{1}=b_{1}, \ldots, z_{i}=b_{i}} \in \mathbb{C}\left[z_{i+1}, \ldots, z_{n}\right]$. For every polynomial set $\boldsymbol{H} \subseteq \mathbb{Q}\left[z_{1}, \ldots, z_{n}\right]$, we define $\boldsymbol{H}(\boldsymbol{b})=\{h(\boldsymbol{b}) \in$ $\left.\mathbb{C}\left[z_{i+1}, \ldots, z_{n}\right] \mid h \in \boldsymbol{H}\right\}$.

Definition 3.1. [32, Definition 4.1] Given $\boldsymbol{H} \subseteq \mathbb{Q}\left[z_{1}, \ldots, z_{n}\right]$, for any $1 \leq i<n$, a subset $\boldsymbol{g}$ of $\boldsymbol{H}$ is a noncomparable subset of $\boldsymbol{H}$ with respect to $z_{i+1}, \ldots, z_{n}$ if

(1) for every $h \in \boldsymbol{H}$, there exists a $g \in \boldsymbol{g}$ such that $\operatorname{lm}_{i}(h)$ is a multiple of $\operatorname{lm}_{i}(g)$, and

(2) for any $g_{1}, g_{2} \in \boldsymbol{g}$, with $g_{1} \neq g_{2}$, the leading monomial $\operatorname{lm}_{i}\left(g_{1}\right)$ is not a multiple of $\operatorname{lm}_{i}\left(g_{2}\right)$, and $\operatorname{lm}_{i}\left(g_{2}\right)$ is not a multiple of $\operatorname{lm}_{i}\left(g_{1}\right)$.

Proposition 3.2. Given $\boldsymbol{H} \subseteq \mathbb{Q}\left[z_{1}, \ldots, z_{n}\right]$, for any $1 \leq i<n$, if the elimination ideal

$$
\langle\boldsymbol{H}\rangle \cap \mathbb{Q}\left[z_{1}, \ldots, z_{i}, z_{i+1}\right]=\langle q\rangle \text { with } \operatorname{deg}\left(q, z_{i+1}\right)>0,
$$

and if $\sqrt{\langle q\rangle}=\langle g\rangle$, then

(1) there exists an affine variety $V \subsetneq \mathbb{C}^{i}$ such that for any $\boldsymbol{b} \in \mathbb{C}^{i} \backslash V$, $\langle\boldsymbol{H}(\boldsymbol{b})\rangle \cap \mathbb{C}\left[z_{i+1}\right]=\langle q(\boldsymbol{b})\rangle$, and

(2) there exists an affine variety $W \subsetneq \mathbb{C}^{i}$ such that for any $\boldsymbol{b} \in \mathbb{C}^{i} \backslash W$, $\sqrt{\langle\boldsymbol{H}(\boldsymbol{b})\rangle \cap \mathbb{C}\left[z_{i+1}\right]}=\langle g(\boldsymbol{b})\rangle$.

Proof. Let $\mathcal{G}$ be a Gröbner basis of $\langle\boldsymbol{H}\rangle$ with respect to the lexicographic order $z_{1}<\cdots<z_{n}$. For any $1 \leq i<n$, let $\mathcal{N}$ be a noncomparable set of $\mathcal{G}$ with respect to $z_{i+1}, \ldots, z_{n}$.

Part (1): If $\langle\boldsymbol{H}\rangle \cap \mathbb{Q}\left[z_{1}, \ldots, z_{i+1}\right]=\langle q\rangle$, then by [18, page 121, Theorem 2], $\mathcal{G} \cap \mathbb{Q}\left[z_{1}, \ldots, z_{i+1}\right]$ is a Gröbner basis of $\langle q\rangle$. So $\mathcal{G} \cap$ $\mathbb{Q}\left[z_{1}, \ldots, z_{i+1}\right]$ contains only one element, say $h$, and hence $h=c \cdot q$ where $c \in \mathbb{Q}$. Also, $\mathcal{G}_{i}=\mathcal{G} \cap \mathbb{Q}\left[z_{1}, \ldots, z_{i}\right]=\emptyset$ since $\operatorname{deg}\left(h, z_{i+1}\right)=$ $\operatorname{deg}\left(q, z_{i+1}\right)>0$, and hence, $\mathcal{V}\left(\mathcal{G}_{i}\right)=\mathbb{C}^{i}$. By [32, Theorem 4.3], there exists $V \subsetneq \mathbb{C}^{i}$ such that for any $\boldsymbol{b} \in \mathbb{C}^{i} \backslash V, \mathcal{N}(\boldsymbol{b})$ is a Gröbner basis of $\langle\boldsymbol{H}(\boldsymbol{b})\rangle$. By [18, page 121, Theorem 2], $\mathcal{N}(\boldsymbol{b}) \cap \mathbb{C}\left[z_{i+1}\right]$ is a Gröbner basis of $\langle\boldsymbol{H}(\boldsymbol{b})\rangle \cap \mathbb{C}\left[z_{i+1}\right]$. Notice that $\mathcal{N}(\boldsymbol{b}) \cap \mathbb{C}\left[z_{i+1}\right]=$ $\{h(\boldsymbol{b})\}$. So, we have $\mathcal{N}(\boldsymbol{b}) \cap \mathbb{C}\left[z_{i+1}\right]=\langle h(\boldsymbol{b})\rangle=\langle q(\boldsymbol{b})\rangle$.

Part (2): If $\sqrt{\langle q\rangle}=\langle g\rangle$, then $\mathcal{V}(q)=\mathcal{V}(g)$. So, for any $\boldsymbol{b} \in \mathbb{C}^{i}$, $\mathcal{V}(q(\boldsymbol{b}))=\mathcal{V}(g(\boldsymbol{b}))$. Hence, we have $\sqrt{\langle q(\boldsymbol{b})\rangle}=\sqrt{\langle g(\boldsymbol{b})\rangle}$. Note that $\langle g\rangle$ is a radical ideal. Then it is a basic fact that there exists an affine variety $V_{1} \subsetneq \mathbb{C}^{i}$ such that for any $\boldsymbol{b} \in \mathbb{C}^{i} \backslash V_{1},\langle g(\boldsymbol{b})\rangle$ is still radical, and hence $\sqrt{\langle q(\boldsymbol{b})\rangle}=\sqrt{\langle g(\boldsymbol{b})\rangle}=\langle g(\boldsymbol{b})\rangle$. By part (1), there exists an affine variety $V_{2} \subsetneq \mathbb{C}^{i}$ such that for any $\boldsymbol{b} \in \mathbb{C}^{i} \backslash V_{2}$, we have the equality in part (1). Let $W=V_{1} \cup V_{2}$. Then for any $\boldsymbol{b} \in \mathbb{C}^{i} \backslash W$, we have the equality in part (2). 
Proposition 3.3. Let $g \in \mathbb{Q}\left[z_{1}, \ldots, z_{n}\right]$. If $g=\Pi_{k=1}^{r} g_{k}^{m_{k}}$, where every $g_{k}$ is irreducible in $\mathbb{Q}\left[z_{1}, \ldots, z_{n}\right]$, and $g_{j} \neq g_{k}$ for any $j \neq k$, then for any $1 \leq i<n$ there exists an infinite subset $\Gamma \subseteq \mathbb{Q}^{i}$ such that for any $\boldsymbol{b} \in \Gamma$ we have $g(\boldsymbol{b})=\Pi_{k=1}^{r} g_{k}(\boldsymbol{b})^{m_{k}}$, where $g_{k}(\boldsymbol{b})$ is irreducible in $\mathbb{Q}\left[z_{i+1}, \ldots, z_{n}\right]$, and $g_{j}(\boldsymbol{b}) \neq g_{k}(\boldsymbol{b})$ for any $j \neq k$.

Proof. Given that $g_{1}, \ldots, g_{r}$ are irreducible, by Hilbert's irreducibility theorem, see e.g., [46, Theorem 1], for any $1 \leq i<n$, there exists an infinite subset $\Theta \subseteq \mathbb{Q}^{i}$ such that for any $\boldsymbol{b} \in W$, $g_{1}(\boldsymbol{b}), \ldots, g_{r}(\boldsymbol{b})$ are irreducible in $\mathbb{Q}\left[z_{i+1}, \ldots, z_{n}\right]$. Now consider an arbitrary pair $g_{j}, g_{k}$ with $j \neq k$ and thus $g_{j} \neq g_{k}$. Without loss of generality, let

$$
W_{j, k}=\left\{\boldsymbol{b} \in \mathbb{C}^{i} \mid g_{j}(\boldsymbol{b})-g_{k}(\boldsymbol{b})=0\right\} .
$$

Obviously, $W_{j, k}$ is an affine variety, which does not equal $\mathbb{C}^{i}$. Then let $\Gamma=\Theta \cup \cup_{k=1}^{r} \cup_{j=1}^{k-1}\left(\mathbb{Q}^{i} \backslash W_{j, k}\right)$, and we are done.

\section{GENERAL ZERO-DIMENSIONAL SYSTEMS}

Throughout the rest of the paper, we always assume that a system of Lagrange likelihood equations is general zero-dimensional; see Definition 4.1. A general zero-dimensional system has a nice structure, see [19, Theorem 6.10]. which leads us to analyze their elimination ideals further. The relation between the Shape Lemma [6] and [19, Theorem 6.10] was discussed in [19].

Definition 4.1 (General Zero-Dimensional System). A polynomial set $\boldsymbol{H}=\left\{h_{1}, \ldots, h_{m}\right\} \subseteq \mathbb{Q}\left[a_{1}, \ldots a_{k}, y_{1}, \ldots, y_{m}\right]$ is called a general zero-dimensional system if there exists an affine variety $V \subsetneq \mathbb{C}^{k}$ such that for any $\boldsymbol{b}=\left(b_{1}, \ldots, b_{k}\right) \in \mathbb{C}^{k} \backslash V$, the equations $h_{1}(\boldsymbol{b})=\cdots=h_{m}(\boldsymbol{b})=0$ satisfy:

(1) the number of complex solutions is a positive constant, denoted by $N(\boldsymbol{H})$;

(2) all complex solutions are distinct;

(3) every pair of distinct complex solutions $\boldsymbol{y}^{*}=\left(y_{1}^{*}, \ldots, y_{m}^{*}\right)$ and $z^{*}=\left(z_{1}^{*}, \ldots, z_{m}^{*}\right)$ it holds that $y_{1}^{*} \neq z_{1}^{*}$.

Proposition 4.2. Consider a general zero-dimensional system $\boldsymbol{H} \subset \mathbb{Q}\left[a_{1}, \ldots, a_{k}, y_{1}, \ldots, y_{m}\right]$. If the elimination ideal

$$
\langle\boldsymbol{H}\rangle \cap \mathbb{Q}\left[a_{1}, \ldots, a_{k}, y_{1}\right]
$$

is principal, then its radical ideal is generated by a polynomial $g \in$ $\mathbb{Q}\left[a_{1}, \ldots, a_{k}, y_{1}\right]$ such that $\operatorname{deg}\left(g, y_{1}\right)=N(\boldsymbol{H})$.

Proof. Let $\mathcal{G}$ be a Gröbner basis of $\langle\boldsymbol{H}\rangle$ with respect to the lexicographic order $a_{1}<\cdots<a_{k}<y_{1}<\cdots<y_{m}$. Since $\boldsymbol{H}$ is general zero-dimensional, by [19, Theorem 6.10], there exists

$$
T_{1}=C_{N} y_{1}^{N}+C_{N-1} y_{1}^{N-1}+\ldots+C_{1} y_{1}+C_{0} \in \mathcal{G},
$$

where $N=N(\boldsymbol{H})$, and $C_{i} \in \mathbb{Q}\left[a_{1}, \ldots, a_{k}\right]$. By [18, page 121 , Theorem 2], $\mathcal{G} \cap \mathbb{Q}\left[a_{1}, \ldots, a_{k}, y_{1}\right]$ is a Gröbner basis of $\langle\boldsymbol{H}\rangle \cap$ $\mathbb{Q}\left[a_{1}, \ldots, a_{k}, y_{1}\right]$. By the hypothesis that $\langle\boldsymbol{H}\rangle \cap \mathbb{Q}\left[a_{1}, \ldots, a_{k}, y_{1}\right]$ is principal, $\mathcal{G} \cap \mathbb{Q}\left[a_{1}, \ldots, a_{k}, y_{1}\right]$ contains only one element. Notice $T_{1} \in \mathcal{G} \cap \mathbb{Q}\left[a_{1}, \ldots, a_{k}, y_{1}\right]$. So, we know $\left\{T_{1}\right\}=\mathcal{G} \cap \mathbb{Q}\left[a_{1}, \ldots, a_{k}, y_{1}\right]$. And hence, $\langle\boldsymbol{H}\rangle \cap \mathbb{Q}\left[a_{1}, \ldots, a_{k}, y_{1}\right]=\left\langle T_{1}\right\rangle$. By [18, page 187, Proposition 12], $\sqrt{\left\langle T_{1}\right\rangle}=\langle g\rangle$, where $g=\frac{T_{1}}{\operatorname{gcd}\left(T_{1}, \frac{\partial T_{1}}{\partial a_{1}}, \ldots, \frac{\partial T_{1}}{\partial a_{k}}, \frac{\partial T_{1}}{\partial y_{1}}\right)}$. Hence, $\operatorname{deg}\left(g, y_{1}\right) \leq \operatorname{deg}\left(T_{1}, y_{1}\right)=N(\boldsymbol{H})$
Below, we prove $\operatorname{deg}\left(g, y_{1}\right) \geq N(\boldsymbol{H})$. By Definition 4.1, there exists an affine variety $V_{1} \subsetneq \mathbb{C}^{k}$ such that for any $\boldsymbol{b} \in \mathbb{C}^{k} \backslash V_{1}, \mathcal{V}(\boldsymbol{H}(\boldsymbol{b}))$ has $N(\boldsymbol{H})$ distinct complex points with distinct $y_{1}$-coordinates. By Proposition 3.2 (2), there exists $V_{2} \subsetneq \mathbb{C}^{k}$ such that for any $\boldsymbol{b} \in \mathbb{C}^{k} \backslash V_{2}$,

$$
\sqrt{\langle\boldsymbol{H}(\boldsymbol{b})\rangle \cap \mathbb{Q}\left[y_{1}\right]}=\langle g(\boldsymbol{b})\rangle .
$$

Let $\boldsymbol{b} \in \mathbb{C}^{k} \backslash\left(V_{1} \cup V_{2}\right)$. Then $g(\boldsymbol{b})=0$ has $N(\boldsymbol{H})$ distinct complex solutions, which are the $y_{1}$-coordinates of points in $\mathcal{V}(\boldsymbol{H}(\boldsymbol{b}))$. So $\operatorname{deg}\left(g, y_{1}\right) \geq \operatorname{deg}\left(g(\boldsymbol{b}), y_{1}\right) \geq N(\boldsymbol{H})$.

\section{ALGORITHM}

Given an algebraic model $\mathcal{M}$, let its Lagrange likelihood equation system be $f=\left\{f_{0}, \ldots, f_{n+s+1}\right\} \subseteq \mathbb{Q}[\boldsymbol{u}, \boldsymbol{p}, \lambda]$. Assuming $\langle f\rangle \cap$ $\mathbb{Q}\left[\boldsymbol{u}, p_{0}\right]$ is principal, we propose a probabilistic algorithm for computing the polynomial $E_{f}$ generating $\sqrt{\langle f\rangle \cap \mathbb{Q}\left[\boldsymbol{u}, p_{0}\right]}$. We simply denote coeff $\left(E_{f}, p_{0}^{i}\right)$ by $A_{i}(\boldsymbol{u})$. Then $E_{\boldsymbol{f}}=\sum_{i=0}^{N} A_{i}(\boldsymbol{u}) p_{0}^{i}$, where by Theorem 2.4, Definition 4.1 and Proposition 4.2, $N$ is the ML-degree of $\mathcal{M}$. First, we highlight a fact:

(F1) $E_{f}$ is homogenous with respect to $\boldsymbol{u}$, and hence each $A_{i}$ is homogenous with the same total degree in $\mathbb{Q}[\boldsymbol{u}]$.

We omit the proof of (F1) since the argument is similar to [40, Proposition 2], which is based on a basic fact implied by (2): for every $\left(\boldsymbol{u}, p_{0}\right) \in \operatorname{proj}_{n+2}(\mathcal{V}(f))$ and for any complex scalar $\gamma \neq 0$, $\left(\gamma \boldsymbol{u}, p_{0}\right)$ is also in $\operatorname{proj}_{n+2}(\mathcal{V}(f))$.

Besides observing (F1), we make the following assumptions to simplify our algorithm:

(A1) Assume $\operatorname{deg}\left(A_{N}, u_{0}\right)=\operatorname{deg}\left(A_{N}\right)$, i.e., $A_{N}$ contains a term $u_{0}^{\operatorname{deg}\left(A_{N}\right)} \in \mathbb{Q}\left[u_{0}\right]$.

(A2) Assume $A_{N}$ is monic with respect to $u_{0}$, which unifies our output polynomial $E_{f}$.

If (A1) does not hold, then we apply an invertible linear change to the parameters $u_{j}$ such that (A1) holds for the new parameters (similar to [40, Algorithm 4]). For instance, obtain new parameters $v_{j}$ as

$$
v_{0}=u_{0}, \text { and } v_{j}=b_{j} u_{j}+u_{0} \text { for } j=1, \ldots, n,
$$

where $b_{j}$ are randomly chosen rational numbers. By [40, Lemmas 1-2], $\operatorname{deg}\left(A_{N}(\boldsymbol{v}), v_{0}\right)$ will be equal to $\operatorname{deg}\left(A_{N}(\boldsymbol{v})\right)$.

Let

$$
\mathcal{S}(\boldsymbol{u})=\sum_{i=0}^{n} u_{i}
$$

The key idea of our algorithm is an observation from experiments: $\mathcal{S}(\boldsymbol{u})$ appears in some coefficients of $E_{\boldsymbol{f}}$ with respect to $p_{0}$. So, we further write

$$
E_{f}\left(\boldsymbol{u}, p_{0}\right)=\sum_{i=0}^{N} A_{i}(\boldsymbol{u}) p_{0}^{i}=\sum_{i=0}^{N} \mathcal{S}(\boldsymbol{u})^{\alpha_{i}} R_{i}(\boldsymbol{u}) p_{0}^{i},
$$

where $R_{i} \in \mathbb{Q}[\boldsymbol{u}] \backslash\langle\mathcal{S}(\boldsymbol{u})\rangle$. In a separate paper, we intend to prove for a general model that at least one $\alpha_{i}$ in (3) is nonzero. The main algorithm has three steps; see Algorithm 1:

Step 1 Compute $N,\left(\alpha_{0}, \ldots, \alpha_{N}\right)$, and the degree of every $u_{j}$ in each $A_{i}$. 

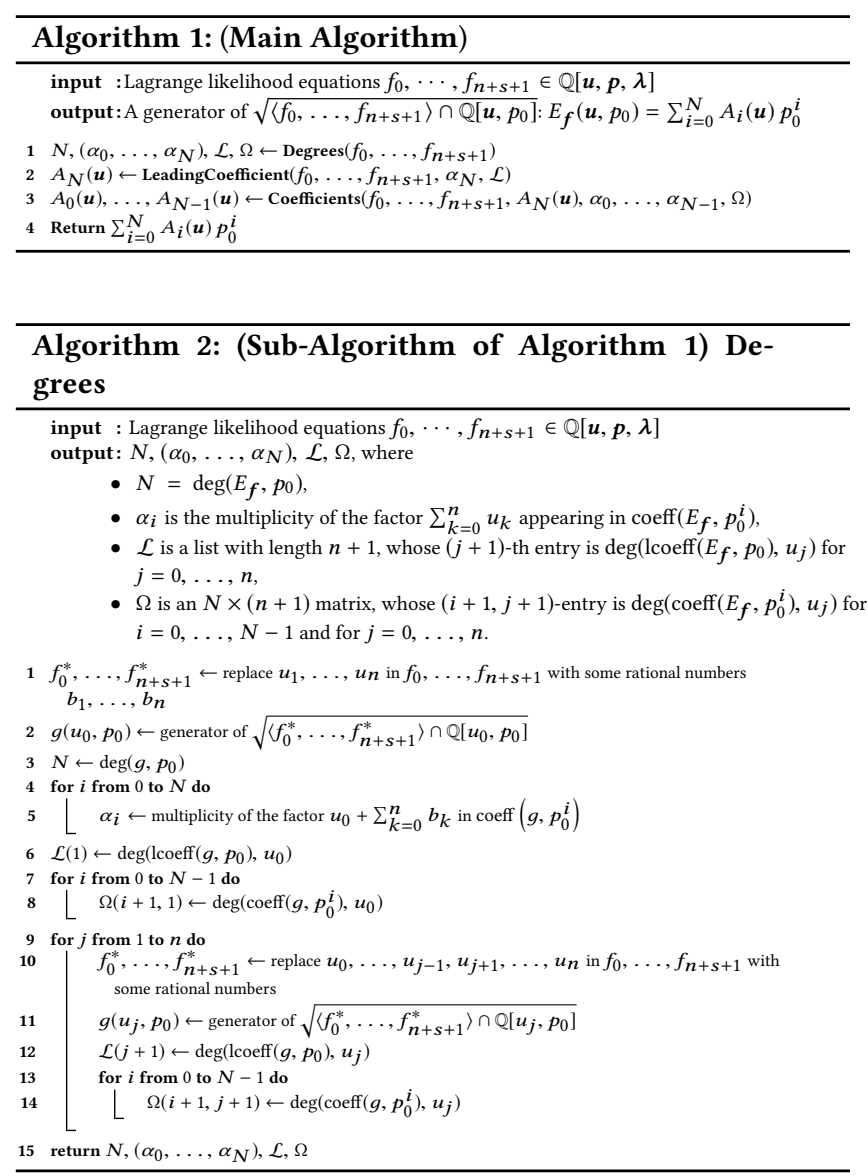

Step 2 Compute the leading coefficient $A_{N}(\boldsymbol{u})$ by interpolating $R_{N}(\boldsymbol{u})$.

Step 3 Compute the coefficients $A_{i}(\boldsymbol{u})$ by interpolating $R_{i}(\boldsymbol{u})$ for $i=0, \ldots, N-1$.

We present the pseudocode in Algorithm 1 and its sub-algorithms Algorithms 2-6, and a running example in Section 5.1. Algorithm 1 is guaranteed to terminate since we only have finite loops. The algorithm is probabilistic, but Propositions 3.2-3.3 guarantee that it provides the correct output for a generical choice of random rational numbers.

\subsection{Running Example}

In this subsection, we illustrate how Algorithm 1 works by the linear model $\mathcal{M}$ below given by a weighted four-sided die [40, Example 1], for which we know the ML-degree is 3:

$$
\mathcal{M}=\mathcal{V}\left(p_{0}+2 p_{1}+3 p_{2}-4 p_{3}\right) \cap \Delta_{3},
$$

where $\Delta_{3}=\left\{\left(p_{0}, p_{1}, p_{2}, p_{3}\right) \in \mathbb{R}_{>0}^{4} \mid p_{0}+p_{1}+p_{2}+p_{3}=1\right\}$. The input Lagrange likelihood equations (2) are

$$
\begin{array}{ll}
f_{0}=p_{0} \lambda_{1}+p_{0} \lambda_{2}-u_{0} & f_{1}=p_{1} \lambda_{1}+2 p_{1} \lambda_{2}-u_{1} \\
f_{2}=p_{2} \lambda_{1}+3 p_{2} \lambda_{2}-u_{2} & f_{3}=p_{3} \lambda_{1}-4 p_{3} \lambda_{2}-u_{3} \\
f_{4}=p_{0}+2 p_{1}+3 p_{2}-4 p_{3} & f_{5}=p_{0}+p_{1}+p_{2}+p_{3}-1
\end{array}
$$
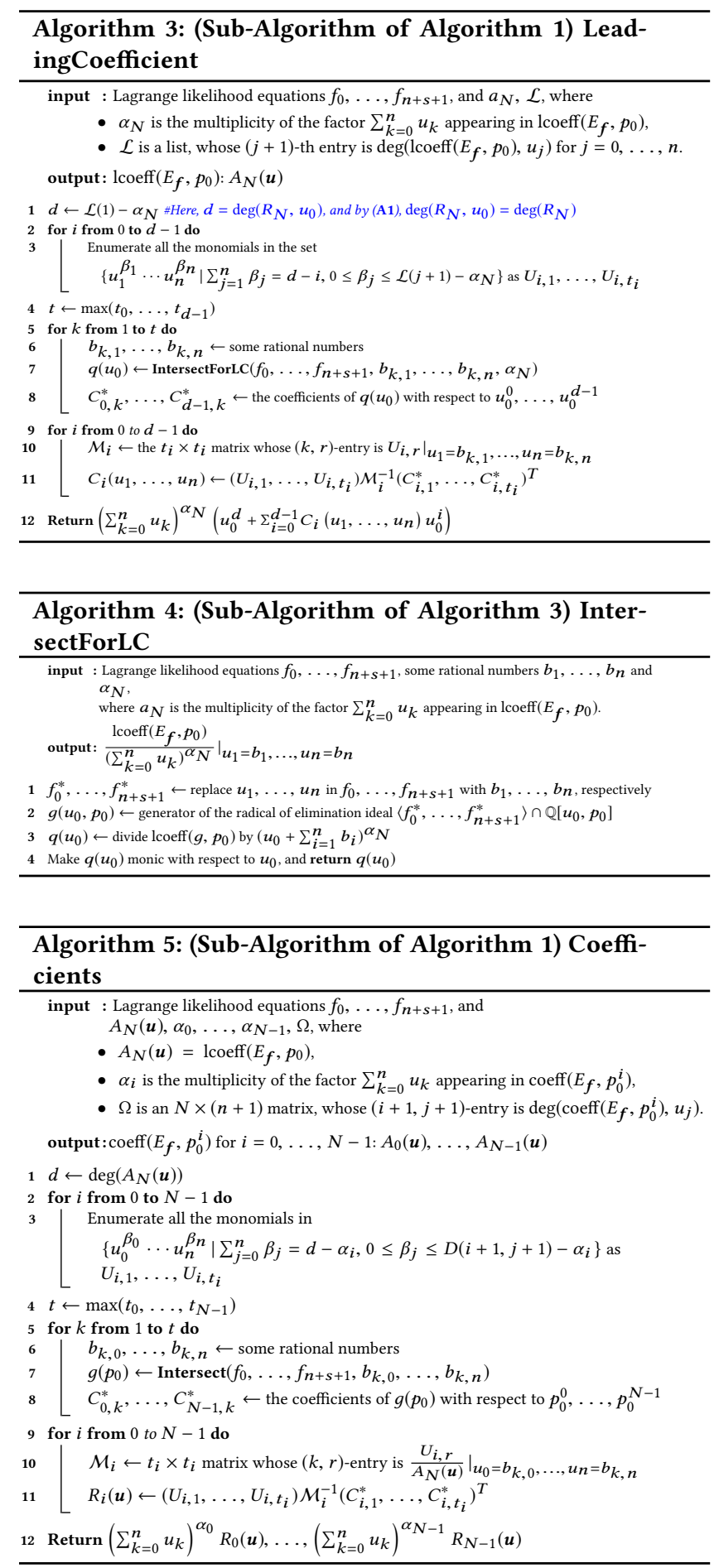

where $p_{0}, p_{1}, p_{2}, p_{3}, \lambda_{1}, \lambda_{2}$ are variables, and $u_{0}, u_{1}, u_{2}, u_{3}$ are parameters. The output is a generator of $\sqrt{\left\langle f_{0}, \ldots, f_{5}\right\rangle \cap \mathbb{Q}\left[\boldsymbol{u}, p_{0}\right]}$. We write the generator as in the form (3).

Step 1. First, we compute $N,\left(\alpha_{0}, \ldots, \alpha_{N}\right)$, and $\operatorname{deg}\left(A_{i}, u_{j}\right)$ for $j=$ $0, \ldots, 3$ and for $i=0, \ldots, N$. For each $u_{j} \neq u_{0}$, substitute $u_{j}=b_{j}$ into $f_{0}, \ldots, f_{5}$, where $b_{j}$ is a random rational number. For instance, 


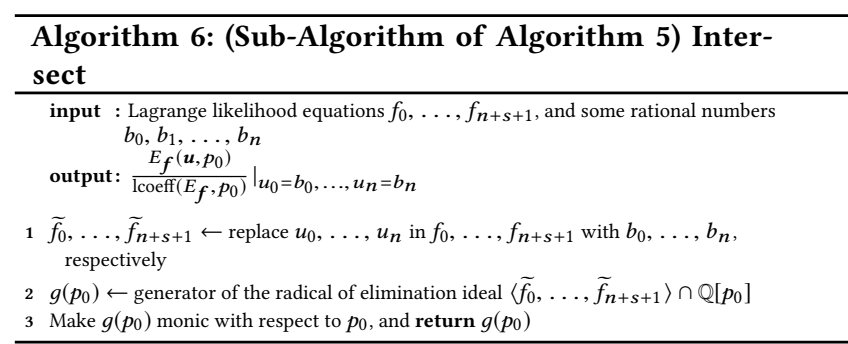

we choose $\boldsymbol{b}=\left(b_{1}, b_{2}, b_{3}\right)=(2,12,7)$. We substitute $u_{j}=b_{j}$, and rename the resulting polynomials as $f_{0}^{*}, \ldots, f_{5}^{*}$. Note that $f_{k}^{*}=$ $f_{k}\left(u_{0}, \boldsymbol{b}, \boldsymbol{p}, \boldsymbol{\lambda}\right)$. We obtain a generator of $\sqrt{\left\langle f_{0}^{*}, \ldots, f_{5}^{*}\right\rangle \cap \mathbb{Q}\left[u_{0}, p_{0}\right]}$ by computing a Gröbner basis: $g^{*}\left(u_{0}, p_{0}\right)=10\left(u_{0}+21\right)^{2} p_{0}^{3}-\left(u_{0}+\right.$ 21) $\left(43 u_{0}+276\right) p_{0}^{2}+2 u_{0}\left(29 u_{0}+396\right) p_{0}-24 u_{0}^{2}$. If $\boldsymbol{b}$ is generic in the parameter space $\mathbb{C}^{3}$, then by Proposition $3.2(2), g^{*}\left(u_{0}, p_{0}\right)=$ $E_{f}\left(u_{0}, b, p_{0}\right)$. So, we have

$$
N=\operatorname{deg}\left(E_{\boldsymbol{f}}\left(\boldsymbol{u}, p_{0}\right), p_{0}\right)=\operatorname{deg}\left(E_{\boldsymbol{f}}\left(u_{0}, \boldsymbol{b}, \boldsymbol{p}_{0}\right), \boldsymbol{p}_{0}\right)=\operatorname{deg}\left(g^{*}, \boldsymbol{p}_{0}\right)=3 .
$$

And, for $i=0, \ldots, N(=3)$, we have

$$
\operatorname{deg}\left(A_{i}(\boldsymbol{u}), u_{0}\right)=\operatorname{deg}\left(A_{i}\left(u_{0}, \boldsymbol{b}\right), u_{0}\right)=\operatorname{deg}\left(\operatorname{coeff}\left(g^{*}, p_{0}^{i}\right), u_{0}\right)=2 .
$$

So, we record $\mathcal{L}(1)=\operatorname{deg}\left(A_{3}, u_{0}\right)=2$ and $\Omega(i+1,1)=\operatorname{deg}\left(A_{i}, u_{0}\right)=$ 2 for $i=0,1,2$. Similarly, we compute the degrees of other parameters, and have

$$
\mathcal{L}=[2,2,2,2] \text {, and } \Omega=\left[\begin{array}{cccc}
2 & 0 & 0 & 0 \\
2 & 1 & 1 & 1 \\
2 & 2 & 2 & 2
\end{array}\right] \text {, }
$$

where $\mathcal{L}(j+1)$ records $\operatorname{deg}\left(A_{3}, u_{j}\right)$, and $\Omega(i+1, j+1)$ records $\operatorname{deg}\left(A_{i}, u_{j}\right)$ for $i=0,1,2$. Notice $\mathcal{S}\left(u_{0}, \boldsymbol{b}\right)=u_{0}+21$. By Proposition 3.3, checking the multiplicity of the factor $u_{0}+21$ in each $\operatorname{coeff}\left(g^{*}, p_{0}^{i}\right)$ for $i=0, \ldots, 3$, we have $\alpha_{0}=\alpha_{1}=0, \alpha_{2}=1$, and $\alpha_{3}=2$.

Step 2. The second step is to recover the leading coefficient $A_{N}(\boldsymbol{u})$. By Step 1, we know $N=3$ and $\alpha_{3}=2$. We write $A_{N}$ as $A_{3}(\boldsymbol{u})=$ $\mathcal{S}(\boldsymbol{u})^{2} R_{3}(\boldsymbol{u})$. By the degrees recored in $\mathcal{L}$, we know the degrees of $u_{0}, u_{1}, u_{2}, u_{3}$ in $A_{3}(\boldsymbol{u})$ are all 2. So, $R_{3}(\boldsymbol{u}) \in \mathbb{Q}$. According to the assumption (A2), $A_{3}(\boldsymbol{u})$ is monic with respect to $u_{0}$. Hence, $R_{3}(\boldsymbol{u})=1$ and $A_{3}(\boldsymbol{u})=\mathcal{S}(\boldsymbol{u})^{2}$.

Step 3. The last step is to interpolate $A_{0}(\boldsymbol{u}), A_{1}(\boldsymbol{u})$ and $A_{2}(\boldsymbol{u})$. As an example, we show how to interpolate $A_{2}(\boldsymbol{u})$ in details. By Step $\mathbf{1}$, we have $\alpha_{2}=1$. So we write $A_{2}(\boldsymbol{u})=\mathcal{S}(\boldsymbol{u}) R_{2}(\boldsymbol{u})$. By the last row of $\Omega$, the degrees of $u_{0}, u_{1}, u_{2}, u_{3}$ in $A_{2}(\boldsymbol{u})$ are 2,2,2,2. Thus, the degrees of $u_{0}, u_{1}, u_{2}, u_{3}$ in $R_{2}(\boldsymbol{u})$ are $1,1,1,1$, respectively. By (F1), $A_{2}$ is homogenous, and we have $\operatorname{deg}\left(A_{2}\right)=\operatorname{deg}\left(A_{3}\right)=2$. So $R_{2}$ is also homogenous, and $\operatorname{deg}\left(R_{2}\right)=\operatorname{deg}\left(A_{2}\right)-\operatorname{deg}(\mathcal{S}(\boldsymbol{u}))=$ 1. Then we can assume $R_{2}(\boldsymbol{u})=\sum_{k=0}^{3} C_{k} u_{k}$, where $C_{k} \in \mathbb{Q}$. In order to determine the four coefficients $C_{k}$, we establish four linear equations by sampling four times. The correctness of this sampling step is guaranteed by Proposition 3.2 (2). We show below how to do the sampling and establish the first linear equation (4) in details. The other equations in (5) are similarly obtained.

For every $u_{j}$, substitute $u_{j}=b_{j}$ into $f_{0}, \ldots, f_{5}$, where $b_{j}$ is a random rational number. For instance, we choose $\boldsymbol{b}=\left(b_{0}, b_{1}, b_{2}, b_{3}\right)=$
$(5,6,11,32)$. We substitute $u_{j}=b_{j}$, and rename the resulting polynomials as $\widetilde{f}_{0}, \ldots, \widetilde{f}_{5}$. Note $\widetilde{f}_{k}=f_{k}(\boldsymbol{b}, \boldsymbol{p}, \boldsymbol{\lambda})$. We compute a generator of $\sqrt{\left\langle\widetilde{f}_{0}, \ldots, \widetilde{f}_{5}\right\rangle \cap \mathbb{Q}\left[p_{0}\right]}$ and make it monic:

$$
\widehat{g}^{(1)}\left(p_{0}\right)=p_{0}^{3}-\frac{7}{5} p_{0}^{2}+\frac{481}{1458} p_{0}-\frac{5}{243} .
$$

By Proposition 3.2 (2), if $\boldsymbol{b}$ is generic in $\mathbb{C}^{4}$, then $\widetilde{g}^{(1)}\left(p_{0}\right)=\frac{E_{\boldsymbol{f}}\left(\boldsymbol{b}, p_{0}\right)}{A_{3}(\boldsymbol{b})}$. So coeff $\left(\widetilde{g}^{(1)}, p_{0}^{2}\right)=\frac{A_{2}(\boldsymbol{b})}{A_{3}(\boldsymbol{b})}$. By the discussion above, we have $A_{2}(\boldsymbol{u})=$ $\mathcal{S}(\boldsymbol{u}) \sum_{k=0}^{3} C_{k} u_{k}$, and by Step $2, A_{3}(\boldsymbol{u})=\mathcal{S}(\boldsymbol{u})^{2}$. So, we obtain

$$
-\frac{7}{5}=\operatorname{coeff}\left(\widetilde{g}^{(1)}, p_{0}^{2}\right)=\frac{A_{2}(\boldsymbol{b})}{A_{3}(\boldsymbol{b})}=\frac{5 C_{0}+6 C_{1}+11 C_{2}+32 C_{3}}{54} .
$$

Similarly, we obtain the other linear equations by samplings:

$$
\begin{aligned}
-\frac{311}{120}=\frac{11 C_{0}+2 C_{1}+3 C_{2}+8 C_{3}}{24} & ,-\frac{244}{115}=\frac{7 C_{0}+2 C_{1}+5 C_{2}+9 C_{3}}{23}, \\
& -\frac{181}{110}=\frac{7 C_{0}+3 C_{1}+13 C_{2}+21 C_{3}}{44} .
\end{aligned}
$$

Solving $C_{0}, \ldots, C_{3}$ from the 4 equations (4)-(5), we have

$$
C_{0}=-\frac{43}{10}, C_{1}=-2, C_{2}=-\frac{3}{2}, C_{3}=-\frac{4}{5},
$$

and hence $A_{2}(\boldsymbol{u})=-\mathcal{S}(\boldsymbol{u})\left(\frac{43}{10} u_{0}+2 u_{1}+\frac{3}{2} u_{2}+\frac{4}{5} u_{3}\right)$. One can similarly interpolate $A_{0}(\boldsymbol{u})$ and $A_{1}(\boldsymbol{u})$. Finally, the output is $E_{\boldsymbol{f}}=\mathcal{S}(\boldsymbol{u})^{2} p_{0}^{3}-$ $\mathcal{S}(\boldsymbol{u})\left(\frac{43}{10} u_{0}+2 u_{1}+\frac{3}{2} u_{2}+\frac{4}{5} u_{3}\right) p_{0}^{2}+\frac{1}{5} u_{0}\left(29 u_{0}+23 u_{1}+21 u_{2}+14 u_{3}\right) p_{0}-$ $\frac{12}{5} u_{0}^{2}$. Also, it is straightforward to check the discriminant of $E_{f}$ with respect to $p_{0}$ is

$4 u_{0}^{2}\left(u_{0}+u_{1}+u_{2}+u_{3}\right)^{2}\left(441 u_{0}^{4}+4998 u_{0}^{3} u_{1}+20041 u_{0}^{2} u_{1}^{2}+33320 u_{0} u_{1}^{3}+19600 u_{1}^{4}-\right.$ $756 u_{0}^{3} u_{2}+20034 u_{0}^{2} u_{1} u_{2}+83370 u_{0} u_{1}^{2} u_{2}+79800 u_{1}^{3} u_{2}-5346 u_{0}^{2} u_{2}^{2}+55890 u_{0} u_{1} u_{2}^{2}+$ $119025 u_{1}^{2} u_{2}^{2}+4860 u_{0} u_{2}^{3}+76950 u_{1} u_{2}^{3}+18225 u_{2}^{4}-1596 u_{0}^{3} u_{3}-11116 u_{0}^{2} u_{1} u_{3}-$ $17808 u_{0} u_{1}^{2} u_{3}+4480 u_{1}^{3} u_{3}+7452 u_{0}^{2} u_{2} u_{3}-7752 u_{0} u_{1} u_{2} u_{3}+49680 u_{1}^{2} u_{2} u_{3}-$ $17172 u_{0} u_{2}^{2} u_{3}+71460 u_{1} u_{2}^{2} u_{3}+27540 u_{2}^{3} u_{3}+2116 u_{0}^{2} u_{3}^{2}+6624 u_{0} u_{1} u_{3}^{2}-4224 u_{1}^{2} u_{3}^{2}-$ $\left.9528 u_{0} u_{2} u_{3}^{2}+15264 u_{1} u_{2} u_{3}^{2}+14724 u_{2}^{2} u_{3}^{2}-1216 u_{0} u_{3}^{3}-512 u_{1} u_{3}^{3}+3264 u_{2} u_{3}^{3}+256 u_{3}^{4}\right)$,

where the last factor is the mixed discriminant of $f_{0}, \ldots, f_{5}$.

\section{COMPUTATIONAL RESULTS}

In this section, we explain the implementation details, and compare the timings of Algorithm 1 and existing methods by testing a list of interesting algebraic models.

\subsection{Implementation}

First, we explain the implementation and experimental details. Testing models, Maple code and computational results are available online via:

https://sites.google.com/site/rootclassification/publications/supplementary-materials/ lle2018.

Software We implemented Algorithm 1 in Maple 2018, where we use the FGb command fgb_gbasis_elim for computing elimination ideals, for instance, in Algorithm 2-Lines 2, 11, Algorithm 4-Line 2 and Algorithm 6-Line 2.

Hardware and System We used a $3.2 \mathrm{GHz}$ Intel Core i5 processor (8 GB of RAM) under OS X 10.9.3.

Testing Models Testing Models are chosen from the literatures $[20,27]$ and have been tested by both standard elimination method and Algorithm 1. 


\subsection{Computing elimination ideals}

We have computed the radical elimination ideals $E_{f}$ for testing models by standard elimination, [40, Algorithm 2] and Algorithm 1. Table 1 compares the timings of the three methods.

Conclusion from Table 1: For smaller models with ML-degree less than 5, computing Gröbner bases directly (standard elimination) is the fastest method; for larger models with ML-degree greater than 5, Algorithm 1 is the fastest. Particularly, comparing columns "Interpolation" and "Algorithm 1", we see the structure of elimination ideals indeed improves the efficiency significantly.

\section{Instruction for Table 1:}

(1) The columns " $\# p_{i}$ " and "ML-Degree" give the number of probability variables $n$ and ML-degree $N$, respectively.

(2) In the column "standard" we record the time to compute the elimination ideal $\langle f\rangle \cap \mathbb{Q}\left[\boldsymbol{u}, p_{0}\right]$. When FGb returned no output until we run out the memory, we record " $\infty$ ".

(3) We record timings of [40, Algorithm 2] and Algorithm 1 in the columns "Interpolation" and "Algorithm 1". Timings in italics font means the computation did not finish within two weeks, but we estimate the sampling timing providing a lower bound; see Example 6.1.

Example 6.1. In Table 1, the estimated total timings for sampling, see Step 3 in Section 5.1, are displayed in italic. We explain how to estimate these timings by the running example in Section 5.1. There are 4 parameters $u_{i}(i=0,1,2,3)$. We know $\operatorname{deg}\left(A_{2}, u_{i}\right)$ are all 2 . Also by (F1) and (A1), $A_{2}$ is homogenous and $\operatorname{deg}\left(A_{2}\right)=\operatorname{deg}\left(A_{2}, u_{0}\right)=2$. So $A_{2}$ is a linear combination of 10 monomials. [40, Algorithm 2] interpolates $A_{2}(\boldsymbol{u})$ directly without any structure, so we need to sample 10 times. However, Algorithm 1 interpolates $R_{2}(\boldsymbol{u})$, which is a factor of $A_{2}(\boldsymbol{u})$ as shown in the running example, so we only need to sample 4 times since there are 4 possible monomials in $R_{2}(\boldsymbol{u})$. We check by Maple the timing for doing sample once in Step 3 is 0.02 second. Then we estimate the timing of sampling in [40, Algorithm 2] and Algorithm 1 are $0.02 \times 10=2$ (seconds) and $0.02 \times 4=0.08$ (second), respectively.

\subsection{Computing discriminants}

Given $E_{f}\left(\boldsymbol{u}, p_{0}\right)$, we can directly compute the discriminant of $E_{f}$ with respect to $p_{0}$, denoted by $\operatorname{discr}\left(E_{f} ; p_{0}\right)$, by eliminating $p_{0}$ from $E_{f}$ and $\frac{\partial E_{f}}{\partial p_{0}}$. We summarize the computational timing of this method for computing discriminants in Table 2. Here, we have a list of remarks on Table 2:

(1) We can not save the large result for Model 6 into a text file. The size of a temporary file when we interrupt the saving process is $32 \mathrm{~GB}$.

(2) For Model 6 and Model 9, the estimated timings for [40, Algorithm 2] to compute $\mathcal{D}_{M J}$ s are 13374 and 454833 days, respectively. Note that $\mathcal{D}_{M J}$ is a factor of $\operatorname{discr}\left(E_{f} ; p_{0}\right)$.

\section{ACKNOWLEDGMENTS}

We thank David A. Cox, Hoon Hong, Anne Shiu, Frank Sottile, and Bernd Sturmfels for their support and advice. TdW was partially supported by the DFG grant WO 2206/1-1. XT was partially supported by the NSF (DMS-1513364, DMS-1752672, and CCF-1708884).

\begin{tabular}{|c|c|c|c|c|c|}
\hline \multirow{2}{*}{ Models } & \multirow{2}{*}{ Degree } & \multirow{2}{*}{ Size } & \multicolumn{3}{|c|}{ Our Method } \\
\cline { 4 - 6 } & & & $E_{\boldsymbol{f}}$ & $\operatorname{discr}\left(E_{\boldsymbol{f}} ; \boldsymbol{p}_{0}\right)$ & Total \\
\hline Model 4 & 110 & $7.5 \mathrm{MB}$ & $782.676 \mathrm{~s}$ & $0.027 \mathrm{~s}$ & $\mathbf{7 8 3} \mathrm{s}$ \\
\hline Model 6 & 342 & $>32 \mathrm{~GB}$ & $14 \mathrm{~d}$ & $11.379 \mathrm{~s}$ & $\mathbf{1 4} \mathrm{d}$ \\
\hline Model 9 & 176 & $8.68 \mathrm{~GB}$ & $2 \mathrm{~d}$ & $81.015 \mathrm{~s}$ & $\mathbf{2} \mathrm{d}$ \\
\hline
\end{tabular}

Table 2: Runtimes for computing discriminants

\section{REFERENCES}

[1] C. Amendola, N. Bliss, I. Burke, C. R. Gibbons, M. Helmer, S. Hoșten, E. D. Nash, J. I. Rodriguez, and D. Smolkin. 2018. The maximum likelihood degree of toric varieties. (2018). Accepted by J. Symb. Comput. Arxiv: 1703.02251.

[2] D. S. Arnon and S. Dennis. 1988. A cluster-based cylindrical algebraic decompoisition algorithm. F. Symb. Comput. 5, 1 (1988), 189-212.

[3] S. Basu, R. Pollack, and M. F. Roy. 1996. On the combinatorial and algebraic complexity of quantifier elimination. Journal of ACM 43, 6 (1996), 1002-1045.

[4] S. Basu, R. Pollack, and M. F. Roy. 1999. Computing roadmaps of semi-algebraic sets on a variety. Fournal of the AMS 3, 1 (1999), 55-82.

[5] S. Basu, R. Pollack, and M. F. Roy. 2006. Algorithms in Real Algebraic Geometry. Springer-Verlag.

[6] E. Becker, M. G. Marinari, T. Mora, and C. Traverso. 1994. The shape of the Shape Lemma. In Proceedings of ISSAC'94. ACM New York, 129-133.

[7] C. W. Brown. 2001. Improved projection for cylindrical algebraic decomposition. 7. Symb. Comput. 32, 5 (2001), 447-465.

[8] C. W. Brown. 2001. Simple CAD construction and its applications. J. Symb. Comput. 31, 5 (2001), 521-547.

[9] C. W. Brown. 2003. QEPCAD B: a program for computing with semi-algebraic sets using CADs. ACM SIGSAM Bulletin 37, 4 (2003), 97-108.

[10] C. W. Brown. 2012. Fast simplifications for Tarski formulas based on monomial inequalities. f. Symb. Comput 47, 7 (2012), 859-882.

[11] C. W. Brown. 2013. Constructing a single open cell in a cylindrical algebraic decomposition. In ISSAC Proceedings of the International Symposium on Symbolic and Algebraic Computation. acm, 133-140.

[12] B. Buchberger. 1965. An algorithm for finding the basis elements of the residue class ring of a zero dimensional polynomial ideal. F. Symb. Comput. 41, 3 (1965), 475-511.

[13] M.-L. G. Buot, S. Hoşten, and D. Richards. 2007. Counting and locating the solutions of polynomial systems of maximum likelihood equations, II: The BehrensFisher problem. Statistica Sinica 17 (2007), 1343-1354.

[14] F. Catanese, S. Hoşten, A. Khetan, and B. Sturmfels. 2006. The maximum likelihood degree. Amer. 7. Math. 128, 3 (2006), 671-697.

[15] C. Chen, J. H. Davemport, J. P. May, M. M. Maza, B. Xia, and R. Xiao. 2010. Triangular decomposition of semi-algebraic systems.. In ISSAC'10 Proceedings of the 35th International Symposium on Symbolic and Algebraic Computation. ACM New York, 187-194.

[16] G. E. Collins. 1975. Quantifier Elimination for the Elementary Theory of Real Closed Fields by Cylindrical Algebraic Decomposition. In Lecture Notes In Computer Science, Vol. 33. Springer-Verlag, Berlin, 134-183.

[17] G. E. Collins and H. Hong. 1991. Cylindrical algebraic decomposition for quntifier elimination. F. Symb. Comput. 12, 3 (1991), 299-328.

[18] D. A. Cox, J. Little, and D. Oshea. 2015. Ideals, varieties, and algorithms: an introduction to computational algebraic geometry and commutative algebra. Springer.

[19] A. Dickenstein, M. P. Millán, A. Shiu, and X. Tang. 2019. Multistationarity in structrued reaction networks. Bulletin of Mathematical Biology. 81, 5 (2019), 1527-1581

[20] M. Drton, B. Sturmfels, and S. Sullivant. 2009. Lectures on algebraic statistics. Springer.

[21] J. C. Faugère. 1999. A new efficient algorithm for computing Gröbner bases (F4). Journal of Pure and Applied Algebra 139, 1 (1999), 61-88.

[22] J. C. Faugère, P. Gianni, D. Lazard, and T. Mora. 1993. Efficient computation of zero-dimensional Gröbner bases by change of ordering. 7. Symb. Comput. 16, 4 (1993), 329-344.

[23] D. Grigoriev. 1988. Complexity of deciding Tarski algebra. f. Symb. Comput. 5, 1-2 (1988), 65-108.

[24] E. Gross, M. Drton, and S. Petrović. 2012. Maximum likelihood degree of variance component models. Electronic Journal of Statistics 6 (2012), 993-1016.

[25] E. Gross and J. I. Rodriguez. 2014. Maximum likelihood geometry in the presence of data zeros. In ISSAC'14 Proceedings of the 39th International Symposium on Symbolic and Algebraic Computation. ACM New York, 232-239.

[26] J. Hauenstein, J. I. Rodriguez, and B. Sturmfels. 2012. Maximum likelihood for matrices with rank constraints. Fournal of Algebraic Statistics (2012). To Appear.

[27] S. Hoşten, A. Khetan, and B. Sturmfels. 2005. Solving the likelihood equations. Foundations of Computational Mathematics 5, 4 (2005), 389-407. 
[28] H. Hong. 1990. An improvement of the projection operator in cylindrical algebraic decomposition. In ISSAC Proceedings of the International Symposium on Symbolic and Algebraic Computation. ACM, 261-264.

[29] H. Hong. 1992. Simple solution formula construction in cylindrical algebraic decomposition based quantifier elimination. In ISSAC Proceedings of the International Symposium on Symbolic and Algebraic Computation. ACM, 177-188.

[30] H. Hong and M. Safey EI Din. 2012. Variant Quantifier Elimination. F. Symb. Comput. 47, 7 (2012), 883-901.

[31] J. Huh and B. Sturmfels. 2014. Likelihood geometry. Number 63-117. Springer International Publishing.

[32] D. Kapur, Y. Sun, and D. K. Wang. 2010. A new algorithm for computing comprehensive Gröbner systems. In ISSAC'10 Proceedings of the 35th International Symposium on Symbolic and Algebraic Computation. 29-36.

[33] D. Lazard and F. Rouillier. 2005. Solving parametric polynomial systems. fournal of Symbolic Computation 42, 6 (2005), 636-667.

[34] S. McCallum. 1988. An improved projection operation for cylindrical algebraic decomposition of three-dimensional space. J. Symb. Comput. 5, 1 (1988), 141-161.

[35] S. McCallum. 1999. On projection in CAD-Based quantifier elimination with equational constrants. In ISSAC Proceedings of the International Symposium on Symbolic and Algebraic Computation. ACM, 145-149.

[36] J. Renegar. 1992. On the computational comlexity and geometry of the first-order theory of the reals. Part I. 7. Symb. Comput. 13, 3 (1992), 255-299.

[37] J. Renegar. 1992. On the computational comlexity and geometry of the first-order theory of the reals. Part II. F. Symb. Comput. 13, 3 (1992), 301-327.

[38] J. Renegar. 1992. On the computational comlexity and geometry of the first-order theory of the reals. Part III. F. Symb. Comput. 13, 3 (1992), 329-352.
[39] J. I. Rodriguez and X. Tang. 2015. Data-Discriminants of Likelihood Equations. In ISSAC'15 Proceedings of the 40th International Symposium on Symbolic and Algebraic Computation. ACM New York, 307-314.

[40] J. I. Rodriguez and X. Tang. 2017. A Probabilistic Algorithm for Computing Data-Discriminants of Likelihood Equations. Journal of Symbolic Computation 83 (2017), 342-364.

[41] M. Safey EI Din and E. Schost. 2003. Polar varieties and computation of one point in each connected component of a smooth real algebraic set. In ISSAC'03 Proceedings of International Symposium on Symbolic and Algebraic Computation. 224-231.

[42] M. Safey EI Din and E. Schost. 2004. Properness defects of projections and computaion of in each connected component of a real algebraic set. Discrete and Computational Geometry 32, 3 (2004), 417-430.

[43] B. Sturmfels. 2002. Solving systems of polynomial equations. In Regional conference series in mathematics. Vol. 97. American Mathematical Society, Providence, R. I.

[44] S. Sullivant. 2018. Algebraic Statistics. Graduate Studies in Mathematics, Vol. 194. American Mathematical Society.

[45] A. Tarski. 1951. A decision method for elementary algebra and geometry. University of California Press. University of California Press. University of California Press.

[46] M. B. Villarino, W. Gasarch, and K. W. Regan. 2018. Hilbert's proof of his irreducibility theorem. The American Mathematical Monthly 125, 6 (2018), 513-530.

[47] L. Yang, X. Hou, and B. Xia. 2001. A complete algorithm for automated discovering of a class of inequality-type theorems. Science in China Series F: Information Sciences 44, 1 (2001), 33-49. 